\title{
Ground State Entropy of Potts Antiferromagnets: Bounds, Series, and Monte Carlo Measurements
}

\author{
Robert Shrock*, Shan-Ho Tsai ${ }^{\dagger}$ \\ Institute for Theoretical Physics \\ State University of New York \\ Stony Brook, N. Y. 11794-3840
}

\begin{abstract}
We report several results concerning $W(\Lambda, q)=\exp \left(S_{0} / k_{B}\right)$, the exponent of the ground state entropy of the Potts antiferromagnet on a lattice $\Lambda$. First, we improve our previous rigorous lower bound on $W(h c, q)$ for the honeycomb (hc) lattice and find that it is extremely accurate; it agrees to the first eleven terms with the large- $q$ series for $W(h c, q)$. Second, we investigate the heteropolygonal Archimedean $4 \cdot 8^{2}$ lattice, derive a rigorous lower bound, on $W\left(4 \cdot 8^{2}, q\right)$, and calculate the large- $q$ series for this function to $O\left(y^{12}\right)$ where $y=1 /(q-1)$. Remarkably, these agree exactly to all thirteen terms calculated. We also report Monte Carlo measurements, and find that these are very close to our lower bound and series. Third, we study the effect of non-nearestneighbor couplings, focusing on the square lattice with next-nearest-neighbor bonds.
\end{abstract}

05.20.-y, 64.60.C, 75.10.H

*email: shrock@insti.physics.sunysb.edu

†email: tsai@insti.physics.sunysb.edu 


\section{INTRODUCTION}

Nonzero ground state disorder and associated entropy, $S_{0} \neq 0$, is an important subject in statistical mechanics; a physical realization is provided by ice, for which $S_{0}=0.82 \pm 0.05$ cal/(K-mole), i.e., $S_{0} / k_{B}=0.41 \pm 0.03$ [1]. A particularly simple model exhibiting ground state entropy without the complication of frustration is the $q$-state Potts antiferromagnet (AF) on a lattice $\Lambda$ for sufficiently large $q$ [2]- [3]. This subject also has a deep connection with graph theory in mathematics [4]- [6] since the zero-temperature partition function of the $q$-state Potts $\mathrm{AF}$ on a lattice $\Lambda$ satisfies $Z(\Lambda, q, T=0)_{P A F}=P(\Lambda, q)$ and hence $S_{0} / k_{B}=\ln W(\Lambda, q)$, where $P(G, q)$ is the chromatic polynomial, expressing the number of ways of coloring the vertices of a graph $G$ with $q$ colors such that no two adjacent vertices have the same color, and

$$
W(\Lambda, q)=\lim _{n \rightarrow \infty} P\left(\Lambda_{n}, q\right)^{1 / n}
$$

where $\Lambda_{n}$ denotes an $n$-vertex lattice of type $\Lambda$ (with appropriate boundary conditions). Given the above connection, it is convenient to express our bounds on the ground state entropy in terms of its exponent, $W(\Lambda, q)$. Recently, we studied the ground state entropy in antiferromagnetic Potts models on various lattices, including both Monte Carlo measurements and rigorous upper and lower bounds [7]- [10]. Here we continue this study. The reader is referred to Refs. [7]- [9] for further background and references.

\section{IMPROVED LOWER BOUND ON $W(h c, q)$ FOR THE HONEYCOMB LATTICE}

In Ref. [9], we derived lower and upper bounds on $W(h c, q)$ for the honeycomb (hc) lattice using a method first applied by Biggs to obtain such bounds for the square lattice [11]. We showed that the upper and lower bounds rapidly approached each other for large $q$ and hence became very restrictive even for moderately large $q$. In particular, we obtained the lower bound, $W(h c, q) \geq W(h c, q)_{\ell^{\prime}}$ for $q \geq 3$, where

$$
W(h c, q)_{\ell^{\prime}}=\frac{(q-1)^{3 / 2}}{q^{1 / 2}}
$$

and found that this was very close to the actual value of $W(h c, q)$, as determined from Monte Carlo measurements [7 9], [12]. (We also obtained similar bounds for the triangular lattice.) Accordingly, in this paper we shall focus on deriving rigorous lower bounds for several lattices $\Lambda$, which again turn out to be very accurate not just as bounds but as approximations for the $W(\Lambda, q)$ functions. In passing, we mention that, using the same methods as in Ref. [9], we 
could also derive rigorous upper bounds for additional lattices; however, we concentrate here on the lower bounds because of their very high accuracy as approximations for the $W(\Lambda, q)$ functions. We begin by sharpening our lower bound for the honeycomb lattice. We picture this lattice as a brick lattice with the long axis of the bricks horizontal, as in Fig. 1(a) of Ref. [9]. We consider a sequence of these lattices with $m(n)$ sites in the horizontal (vertical) direction, denoted $(h c)_{m \times n}$. In the thermodynamic limit the boundary conditions do not affect the bound, so for technical convenience, we take free (periodic) boundary conditions along the horizontal (vertical) directions. Further, to maintain the bipartite nature of the lattice and avoid frustration, we take $n$ even. Our bounds in Ref. [9] were obtained by taking the vectors of color configurations to refer to the vertical lines; here we take them to refer to horizontal lines $L$. The number of allowed $q$-colorings of this line of vertices is $\mathcal{N}=P\left(L_{m}, q\right)=q(q-1)^{m-1}$. One can then associate with two adjacent horizontal lines of sites $L, L^{\prime}$, forming one layer of bricks, an $\mathcal{N} \times \mathcal{N}$ dimensional symmetric coloring matrix $T$ with entries $T_{L, L^{\prime}}=1$ or 0 if the $q$-colorings of these lines are or are not compatible. Here, by compatible $q$-colorings we mean colorings that satisfy the constraint that no two adjacent vertices have the same color. Then (for fixed $m, n) P\left((h c)_{m \times n}, q\right)=\operatorname{Tr}\left(T^{n}\right)$. Since $T$ is a nonnegative matrix, one can apply the Perron-Frobenius theorem 13] to conclude that $T$ has a real positive maximal eigenvalue $\lambda_{\max , n}(q)$. Hence, for fixed $m$,

$$
\lim _{n \rightarrow \infty} \operatorname{Tr}\left(T^{n}\right)^{1 /(m n)} \rightarrow \lambda_{\max }^{1 / m}
$$

so that

$$
W(\Lambda, q)=\lim _{m \rightarrow \infty} \lambda_{\max }^{1 / m}
$$

Denote the column sum $\kappa_{j}(T)=\sum_{i=1}^{\mathcal{N}} T_{i j}$ (equal to the row sum $\rho_{j}(T)=\sum_{i=1}^{\mathcal{N}} T_{j i}$ since $T^{T}=T$ ) and $S(T)=\sum_{i, j=1}^{\mathcal{N}} T_{i j}$; note that $S(T) / \mathcal{N}$ is the average row (column) sum. Combining the bounds for a general nonnegative $\mathcal{N} \times \mathcal{N}$ matrix $A$, 13

$$
\min \left\{\gamma_{j}(A)\right\} \leq \lambda_{\max }(A) \leq \max \left\{\gamma_{j}(A)\right\}, \quad \text { for } \quad \gamma_{j}=\kappa_{j} \quad \text { or } \quad \rho_{j}
$$

with the ( $\nu=1$ case of the ) more restrictive lower bound applicable to a symmetric nonnegative matrix [14],

$$
\left[\frac{S\left(T^{\nu}\right)}{\mathcal{N}}\right]^{1 / \nu} \leq \lambda_{\max }, \quad \text { for } \quad \nu=1,2, \ldots
$$

we have

$$
\frac{S(T)}{\mathcal{N}} \leq \lambda_{\max }(T) \leq \max \left\{\kappa_{j}(T)\right\}
$$


The sum $S(T)$ is given by the chromatic polynomial for the brick layer of length $m$, hence containing $2 m$ vertices, which we calculate to be

$$
P\left((C h)_{6,2 m}, q\right)=q(q-1) D_{6}(q)^{(m-1) / 2}
$$

where the notation [10] $(C h)_{k, 2 m}$ means a $2 m$-vertex chain of $k$-sided polygons, with each adjacent pair of $k$-gons intersecting along one common edge, and

$$
D_{k}(q)=\sum_{s=0}^{k-2}(-1)^{s}\left(\begin{array}{c}
k-1 \\
s
\end{array}\right) q^{k-2-s}
$$

Taking the $m \rightarrow \infty$ limit and applying the $\nu=1$ case of (2.5), we thus obtain the lower bound $W(h c, q) \geq W(h c, q)_{\ell}$ for $q \geq 3$ [12, where

$$
W(h c, q)_{\ell}=\frac{D_{6}(q)^{1 / 2}}{q-1}=\frac{\left(q^{4}-5 q^{3}+10 q^{2}-10 q+5\right)^{1 / 2}}{q-1}
$$

For $q \geq 4$, the difference between the lower bounds (2.1) and (2.9) decreases rapidly toward zero. The corresponding reduced $W$ functions $W_{r}(h c, q)=q^{-1} W(h c, q)$ have large- $q$ Taylor series which coincide up to order $O\left(q^{-4}\right)$ :

$$
q^{-1} W(h c, q)_{\ell}=1-\frac{3}{2} q^{-1}+\frac{3}{8} q^{-2}+\frac{1}{16} q^{-3}+\frac{3}{128} q^{-4}+\frac{131}{256} q^{-5}+O\left(q^{-6}\right)
$$

and

$$
q^{-1}\left(W(h c, q)_{\ell}-W(h c, q)_{\ell^{\prime}}\right)=\frac{1}{2} q^{-5}+O\left(q^{-6}\right)
$$

Since the quantity $y=1 /(q-1)$ is a natural variable for a large- $q$ expansion, it is also useful to compare an expansion of our new lower bound with a small- $y$ series. For a lattice $\Lambda$, this series has the form [15]- 17]

$$
W(\Lambda, q)=q\left(1-q^{-1}\right)^{\zeta / 2} \bar{W}(\Lambda, y)
$$

where $\zeta$ is the lattice coordination number, and

$$
\bar{W}(\Lambda, y)=1+\sum_{n=1}^{\infty} w_{n} y^{n}, \quad y=\frac{1}{q-1}
$$

Analogously, for the expansion of our lower bound, we define the reduced lower bound function $\bar{W}(\Lambda, y)_{\ell}$ via

$$
W(\Lambda, q)_{\ell}=q\left(1-q^{-1}\right)^{\zeta / 2} \bar{W}(\Lambda, y)_{\ell}
$$


From eq. (2.9), we find the very simple result

$$
\bar{W}(h c, y)_{\ell}=\left(1+y^{5}\right)^{1 / 2}
$$

Expanding this in a Taylor series in $y$, we have

$$
\bar{W}(h c, y)_{\ell}=1+\frac{1}{2} y^{5}-\frac{1}{8} y^{10}+\frac{1}{16} y^{15}+O\left(y^{20}\right)
$$

Although as a lower bound, this need not, a priori, agree with terms in the small-y Taylor series expansion of the actual function $\bar{W}(h c, y)$, we find that, remarkably, it does agree all the way up to $O\left(y^{10}\right)$, i.e., for the first eleven terms. The small-y Taylor series expansion of $\bar{W}(h c, y)$, calculated to order $O\left(y^{5}\right)$ in Ref. [16] and to $O\left(y^{18}\right)$ in Ref. [17], is:

$$
\bar{W}(h c, y)_{\ell}=1+\frac{1}{2} y^{5}-\frac{1}{8} y^{10}+y^{11}+O\left(y^{12}\right)
$$

To show how close our lower bound is to the actual function $W(h c, q)$, we give a comparison in Table 1 for $q=3$ through $q=10$. We determined the values of $W(h c, q)$ by Monte Carlo measurements, as discussed in detail in our previous Refs. [7] and [8]. We also include a comparison with our slightly less stringent lower bound (2.1). Evidently, both lower bounds are quite close to the actual measurements, even for $q$ as low as 3 . In particular, the sharper lower bound (2.9) provides an extremely good approximation to the actual function $W(h c, q)$ for $q \geq 3$.

TABLE I. Comparison of lower bounds for $W(h c, q)$ and ratios of bounds to Monte Carlo measurements for $3 \leq q \leq 10$. The estimated uncertainty from the MC measurements for the entries in the $W(h c, q)_{\ell} / W(h c, q)_{M C}$ and $W(h c, q)_{\ell^{\prime}} / W(h c, q)_{M C}$ entries is $3 \times 10^{-4}$.

\begin{tabular}{cccccc}
\hline \hline$q$ & $W(h c, q)_{M C}$ & $W(h c, q)_{\ell}$ & $\frac{W(h c, q)_{\ell}}{W(h c, q)_{M C}}$ & $W(h c, q)_{\ell^{\prime}}$ & $\frac{W(h c, q)_{\ell^{\prime}}}{W(h c, q)_{M C}}$ \\
\hline 3 & $1.6600(5)$ & 1.6583124 & 0.99898 & 1.6329932 & 0.98373 \\
4 & $2.6038(7)$ & 2.6034166 & 0.99985 & 2.5980762 & 0.99780 \\
5 & $3.5796(10)$ & 3.5794553 & 0.99996 & 3.5777088 & 0.99947 \\
6 & $4.5654(15)$ & 4.5650849 & 0.99993 & 4.56435465 & 0.99977 \\
7 & $5.5556(17)$ & 5.5552778 & 0.99994 & 5.5549206 & 0.99988 \\
8 & $6.5479(20)$ & 6.5480952 & 1.00003 & 6.5479004 & 1.00000 \\
9 & $7.5424(22)$ & 7.5425874 & 1.00002 & 7.5424723 & 1.00001
\end{tabular}




\section{III. $4 \cdot 8^{2}$ LATTICE}

Another interesting question in statistical mechanics and graph theory is the Potts AF ground state entropy and the related asymptotic behavior of the chromatic polynomial on heteropolygonal lattices, i.e. Archimedean lattices [18 involving tiling of the plane by more than one regular polygon such that all vertices are equivalent. In standard mathematical notation [18,19], an Archimedean lattice is defined by the set $p_{1} \cdot p_{2} \cdots p_{n}$ of polygons that one traverses in a circuit around a vertex (site) of the lattice. For a homopolygonal lattice, these are all the same (e.g., in this notation, the square, honeycomb, and triangular lattices are $4^{4}, 6^{3}$, and $\left.3^{6}\right)$. We consider here the $4 \cdot 8^{2}$ lattice.

\section{A. Lower Bound on $W\left(4 \cdot 8^{2}, q\right)$}

It is convenient to represent the $4 \cdot 8^{2}$ lattice as a generalized brick lattice, containing two types of bricks at each horizontal level: square bricks and rectangular ones with (length,height) $=(3,1)$. In the next layer up, a square brick is located in the center above a $(3,1)$ brick, and a $(3,1)$ brick is located with its center above a square brick, and so forth for successive levels. Using the same general method as before, we construct a coloring matrix defined between adjacent horizontal rows of bricks and obtain the lower bound $W\left(4 \cdot 8^{2}, q\right) \geq W\left(4 \cdot 8^{2}, q\right)_{\ell}$ for $q \geq 3$ [12], where

$$
\begin{aligned}
W\left(4 \cdot 8^{2}, q\right)_{\ell} & =\frac{\left[D_{4}(q) D_{8}(q)\right]^{1 / 4}}{q-1} \\
& =\frac{\left[\left(q^{2}-3 q+3\right)\left(q^{6}-7 q^{5}+21 q^{4}-35 q^{3}+35 q^{2}-21 q+7\right)\right]^{1 / 4}}{q-1}
\end{aligned}
$$

Dividing by the asymptotic behavior as before [9], this has the large- $q$ expansion

$$
q^{-1} W\left(4 \cdot 8^{2}, q\right)_{\ell}=1-\frac{3}{2} q^{-1}+\frac{3}{8} q^{-2}+\frac{5}{16} q^{-3}+\frac{51}{128} q^{-4}+O\left(q^{-5}\right)
$$

The corresponding reduced function $\bar{W}\left(4 \cdot 8^{2}, y\right)$ is

$$
\begin{aligned}
\bar{W}\left(4 \cdot 8^{2}, y\right)_{\ell} & =\left(1+y^{3}+y^{7}+y^{10}\right)^{1 / 4}=\left[\left(1+y^{3}\right)\left(1+y^{7}\right)\right]^{1 / 4} \\
& =(1+y)^{1 / 2}\left[\left(1-y+y^{2}\right)\left(1-y+y^{2}-y^{3}+y^{4}-y^{5}+y^{6}\right)\right]^{1 / 4}
\end{aligned}
$$




\section{B. Large- $q$ Series Expansion}

Using standard methods [15, we have calculated the large $q$ expansion, i.e., the small-y expansion, of the reduced function $\bar{W}\left(4 \cdot 8^{2}, y\right)$. We find

$$
\bar{W}\left(4 \cdot 8^{2}, y\right)=1+\frac{1}{4} y^{3}-\frac{3}{2^{5}} y^{6}+\frac{1}{4} y^{7}+\frac{7}{2^{7}} y^{9}+\frac{1}{2^{4}} y^{10}-\frac{77}{2^{11}} y^{12}+O\left(y^{13}\right)
$$

Next, in order to assess the accuracy for large $q$ of our lower bound, we have expanded the corresponding reduced lower bound function $\bar{W}\left(4 \cdot 8^{2}, y\right)_{\ell}$, eq. (3.3) in a series in $y$. Remarkably, the small-y series expansion of our lower bound agrees to $O\left(y^{12}\right)$, i.e. to all thirteen terms that we have calculated, with the small $y$ expansion of the true $\bar{W}\left(4 \cdot 8^{2}, y\right)$ function in (3.4) ! This shows that for even moderately large $q$, our lower bound $W\left(4 \cdot 8^{2}, q\right)_{\ell}$ actually provides an extremely accurate approximation to the true function $W\left(4 \cdot 8^{2}, q\right)$. Of course, it is straightforward to calculate the series (3.4) to higher order in $y$, and at $O\left(y^{13}\right)$ or above the series for $\bar{W}\left(4 \cdot 8^{2}, y\right)$ and $\bar{W}\left(4 \cdot 8^{2}, y\right)_{\ell}$ could differ; nevertheless, the agreement to $O\left(y^{12}\right)$ is already quite striking.

\section{Monte Carlo Measurements}

To get further information on $W\left(4 \cdot 8^{2}, q\right)$, we have carried out Monte Carlo (MC) measurements of the Potts AF ground state entropy $S_{0}$ and hence obtained $W=\exp \left(S_{0} / k_{B}\right)$, for $3 \leq q \leq 10$. Our methods are similar to those used in our previous works [7]- [8]. We present our results in Table 2, including comparisons with our rigorous lower bound and our series calculation to $O\left(y^{12}\right)$ (to which order they coincide). Evidently, the agreement is extremely good, even for $q$ as low as 3 .

TABLE II. Exponential of ground state entropy, from Monte Carlo measurements, and comparison with lower bound, for the Potts antiferromagnet with $4 \leq q \leq 10$ on the $4 \cdot 8^{2}$ lattice. The estimated uncertainty from the MC measurements for the entries in the $W\left(4 \cdot 8^{2}, q\right)_{\ell} / W\left(4 \cdot 8^{2}, q\right)_{M C}$ column is $3 \times 10^{-4}$.

\begin{tabular}{ccc}
\hline \hline$q$ & $W\left(4 \cdot 8^{2}, q\right)_{M C}$ & $\frac{W\left(4 \cdot 8^{2}, q\right)_{\ell}}{W\left(4 \cdot 8^{2}, q\right)_{M C}}$ \\
\hline 3 & $1.68575(60)$ & 0.99959 \\
4 & $2.62226(75)$ & 0.99994
\end{tabular}




\section{SQUARE LATTICE WITH DIAGONAL NEXT-NEAREST-NEIGHBOR COUPLINGS}

An interesting question concerns the effect of non-nearest-neighbor interactions on the ground-state entropy of the Potts antiferromagnet and the corresponding $W(\Lambda, q)$ function. We explore this question here by considering the Potts AF on a square lattice with nextnearest-neighbor couplings along both diagonals, defined by the Hamiltonian

$$
\mathcal{H}=-\sum_{n, n^{\prime}} J_{n n^{\prime}} \delta_{\sigma_{n}, \sigma_{n^{\prime}}}
$$

where $J_{n, n^{\prime}}=J<0$ if the sites $n$ and $n^{\prime}$ are nearest neighbors along a row or column, and $J_{n, n^{\prime}}=J_{d}<0$ if $n$ and $n^{\prime}$ are diagonally opposite sites on a given square of the lattice (with each such pair counted only once in the sum (4.1)). We further define $K=\beta J$ and $K_{d}=\beta J_{d}$ and consider the $T \rightarrow 0$ limit, so that the color on each site $n$ is required to be different from the colors of each of its nearest neighbors on the rows and columns of the lattice, and also each of its next-nearest neighbors along the diagonals of the lattice. Then $Z\left((s q)_{d}, q, T=0\right)_{P A F}=P\left((s q)_{d}, q\right)$, where $(s q)_{d}$ denotes the nonplanar graph formed from a square lattice by adjoining bonds connecting diagonally opposite sites on each square [20]. Note that the $(s q)_{d}$ lattice has coordination number $\zeta=8$ and girth $\gamma=3$, where "girth" $\gamma$ of a graph $G$ denotes the length of the shortest circuit on $G$. We find that the chromatic number of this lattice (for free boundary conditions or periodic boundary conditions that preserve the bipartite structure of the underlying square lattice before inclusion of diagonal bonds)

$$
\chi\left((s q)_{d}\right)=4
$$

$(\chi(G)$ is defined in footnote [12].) These are to be compared with $\zeta=\gamma=4$ and $\chi(s q)=2$ for the square lattice. From our earlier discussion, one thus has $W\left((s q)_{d}, q\right) \leq W(s q, q)$. Indeed, we find that there is an especially strong difference with respect to the square lattice for the case $q=\chi\left((s q)_{d}\right)=4$. Here, we calculate that for a $(s q)_{d}$ lattice $\Lambda_{L_{1} \times L_{2}}$ of length $L_{1}$ in the $x$ direction and $L_{2}$ in the $y$ direction,

$$
P\left(\Lambda_{L_{1} \times L_{2}}, 4\right) \sim 2^{L_{1}+L_{2}}
$$

in the thermodynamic limit. Although this diverges, it does not diverge rapidly enough to yield a finite entropy. With the ordering of limits

$$
W\left(\Lambda, q_{s}\right) \equiv \lim _{n \rightarrow \infty} \lim _{q \rightarrow q_{s}} P(G, q)^{1 / n}
$$


as discussed in Ref. [8], we thus obtain the exact result

$$
W\left((s q)_{d}, q=4\right)=1 \quad \text { i.e., } \quad S_{0}\left((s q)_{d}, q=4\right)=0
$$

This may be compared with the value for the usual square lattice, for which $W(s q, 4)=$ $2.3370(7)$ [8], corresponding to the nonzero ground state entropy $S_{0}(s q, 4)=0.8489(3)$. (Indeed, even without any detailed calculation, one knows that $S_{0}(s q, 4)$ is nonzero from the elementary rigorous lower bound on a bipartite lattice, $S_{0}\left(\Lambda_{\text {bip. }}, q\right)>(1 / 2) \ln (q-1)$.)

TABLE III. Exponential of ground state entropy, from Monte Carlo measurements, and comparison with lower bound, for the Potts antiferromagnet with $5 \leq q \leq 10$ on the square lattice with diagonal next-nearest-neighbor couplings. The estimated uncertainty from the MC measurements in the entries for $W\left(s q_{d}, q\right)_{\ell} / W\left(s q_{d}, q\right)_{M C}$ is $1 \times 10^{-3}$.

\begin{tabular}{lccr}
\hline \hline$q$ & $W\left(s q_{d}, q\right)_{M C}$ & $\frac{W\left(s q_{d}, q\right)_{\ell}}{W\left(s q_{d}, q\right)_{M C}}$ & $W(s q, q)_{M C}$ \\
\hline 5 & $1.5781(16)$ & 0.95051 & $3.2510(10)$ \\
6 & $2.4460(24)$ & 0.98119 & $4.2003(12)$ \\
7 & $3.3660(33)$ & 0.99029 & $5.1669(15)$ \\
8 & $4.3093(43)$ & 0.99453 & $6.1431(20)$ \\
9 & $5.2680(53)$ & 0.99658 & $7.1254(22)$ \\
10 & $6.2363(62)$ & 0.99774 & $8.1122(25)$ \\
\hline \hline
\end{tabular}


Using the same type of coloring matrix method as above, we obtain the rigorous lower bound $W\left((s q)_{d}, q\right) \geq W\left((s q)_{d}, q\right)_{\ell}$, where

$$
W\left((s q)_{d}, q\right)_{\ell}=\frac{(q-2)(q-3)}{q-1} \quad \text { for } \quad q \geq 4
$$

For large $q$, we have the Taylor series expansion

$$
q^{-1} W\left(s q_{d}, q\right)_{\ell}=1-4 q^{-1}+2\left(q^{-2}+q^{-3}+q^{-4}+O\left(q^{-5}\right)\right)
$$

From eq. (2.14) we have

$$
\bar{W}\left(s q_{d}, y\right)_{\ell}=(1-y)(1-2 y)(1+y)^{3}
$$

The MC measurements are given in Table 1. For comparison, we include also our MC measurements of $W(s q, q)$ [8]. The lower bound (4.6) steadily approaches the measured values $W\left((s q)_{d}, q\right)$ as $q$ increases and is quite close to them for $q$ greater than about 6 . The inequality $W\left((s q)_{d}, q\right) \leq W(s q, q)$ is also evident.

This research was supported in part by the NSF grant PHY-93-09888.

[1] W. F. Giauque and J. W. Stout, J. Am. Chem. Soc. 58, 1144 (1936); L. Pauling, The Nature of the Chemical Bond (Cornell Univ. Press, Ithaca, 1960), p. 466.

[2] R. B. Potts, Proc. Camb. Phil. Soc. 48, 106 (1952).

[3] C. M. Fortuin and P. W. Kasteleyn, Physica 57, 536 (1972).

[4] W. T. Tutte Graph Theory, vol. 21 of Encyclopedia of Mathematics and its Applications, ed. Rota, G. C. (Addison-Wesley, New York, 1984).

[5] R. C. Read and W. T. Tutte, "Chromatic Polynomials", in Selected Topics in Graph Theory, 3, eds. L. W. Beineke and R. J. Wilson (Academic Press, New York, 1988).

[6] N. Biggs, "Algebraic Graph Theory", 2nd ed. (Cambridge, U.K., Cambridge Univ. Press, 1st ed. 1974, 2nd ed. 1993). 
[7] R. Shrock and S.-H. Tsai, J. Phys. A 30, 495 (1997).

[8] R. Shrock and S.-H. Tsai, Phys. Rev. E55, 5165 (1997).

[9] R. Shrock and S.-H. Tsai, Phys. Rev. E55, 6791 (1997).

[10] R. Shrock and S.-H. Tsai, preprints ITP-SB-97-11,12, Phys. Rev. E, in press.

[11] N. L. Biggs, Bull. London Math. Soc. 9, 54 (1977).

[12] We comment on the case $q=2$ here. A bipartite lattice $\Lambda_{b i p}$. has chromatic number $\chi\left(\Lambda_{b i p}.\right)=2$, where $\chi(G)$ is defined as the minimum integer value of $q$ that allows a $q$-coloring of $G$ such that any two adjacent vertices have different colors. In the present work, the honeycomb and $4 \cdot 8^{2}$ lattices are bipartite. In general, there are two ways to color a bipartite lattice with $q=2$ colors (provided that the boundary conditions maintain the bipartite property). For $q=2$, one does not need any bounds since one has an exact result: in the subscript notation of Ref. [8], $W\left(\Lambda_{b i p .}, 2\right)_{D_{n q}}=\lim _{n \rightarrow \infty} \lim _{q \rightarrow 2} P\left(\left(\Lambda_{b i p .}\right)_{n}, q\right)^{1 / n}=1$; equivalently stated, the $q=2$ Potts AF (Ising model) on $\Lambda_{b i p}$. has no ground state entropy.

[13] See, e.g., P. Lancaster and M. Tismenetsky, The Theory of Matrices, with Applications (New York, Academic Press, 1985); H. Minc, Nonnegative Matrices (New York, Wiley, 1988).

[14] D. London, Duke Math. J. 33, 511 (1966).

[15] J. F. Nagle, J. Combin. Theory 10, 42 (1971).

[16] G. A. Baker, Jr., J. Combin. Theory 10, 217 (1971).

[17] D. Kim and I. G. Enting, J. Combin. Theory, B 26, 327 (1979). Note that the function denoted $\bar{W}$ for the honeycomb lattice in this reference is implicitly defined per 2-cell, not per site, and hence is equal to $\bar{W}(h c, y)^{2}$.

[18] Grünbaum, B. and Shephard, G. 1989 Tilings and Patterns: an Introduction (Freeman, New 
York, 1989).

[19] V. Matveev and R. Shrock, J. Phys. A 28, 5235 (1995).

[20] This nonplanar graph differs from the (planar) union jack lattice in that the diagonals do not form a further vertex in the middle of each square. 